\title{
Exhaled NO may predict loss of asthma control: the effect of concomitant allergic rhinitis
}

\section{To the Editors:}

We read with great interest the recent article by MichiLs et al. [1] demonstrating that sequential changes in exhaled NO fraction $(F \mathrm{eNO})$ are associated with asthma control, even in smokers who present lower levels of FeNO compared with nonsmokers. Besides tobacco smoking, we have recently shown that a significant confounding factor in the diagnosis of asthma using FeNO is the presence of allergic rhinitis, since patients with allergic rhinitis have been found to have elevated FeNO levels [2]. This effect may well be attributed to the underlying atopic status of those patients, since elevated FeNO has been shown to be associated with a phenotype characterised by atopy and increased airway responsiveness [3]. In the present study we attempted to evaluate whether the coexistence of allergic rhinitis may affect the validity of sequential changes of $\mathrm{FeNO}$ in predicting control in asthmatic patients.

We performed a post hoc analysis of a prospectively collected database of all consecutive patients evaluated in the Asthma Clinic of the Respiratory Medicine Dept, University of Thessaly Medical School (Larissa, Greece), between June 2007 and February 2009. Of the 259 patients in the database, we excluded 82 smokers, 26 patients with difficult-to-treat asthma in whom total control was never achieved during that time period, and 41 patients who were not seen at least twice. Patients were treated according to Global Initiative for Asthma (GINA) guidelines and were all receiving inhaled corticosteroids. All patients were submitted to FeNO measurements using a portable analyser (NiOX MiNO; Aerocrine, Solna, Sweden) as previously described [2] and to spirometry with a dry spirometer (KoKo Legend; Ferraris Respiratory Ltd, Hertford, UK). The assessment of asthma control was based on the evaluation of an experienced physician (K. Kostikas) according to the GINA guidelines and the use of the original Asthma Control Questionnaire (ACQ) [4]. The diagnosis of allergic rhinitis was based on history and appropriate assessment of atopic status, as previously described [5].

Comparisons among groups at baseline were performed with one-way ANOVA with Bonferroni post hoc tests. Comparisons of FeNO levels between the two visits were performed using Wilcoxon signed rank tests. For the assessment of the performance of $F$ eNO percentage changes compared to baseline $(\Delta F \mathrm{eNO} \%)$ in the identification of loss of control, receiver operating characteristics (ROC) curves were created by plotting sensitivity against 100 -specificity. The area under the ROC curve (AUC) with 95\% confidence intervals (CI) and its difference from 0.5 were calculated. Additionally, sensitivities, specificities, and positive and negative predictive values (PPV and NPV, respectively) were calculated for specific cut-off points. Statistical analysis was performed with GraphPad Prism 5 (GraphPad Software Inc, La Jolla, CA, USA) and MedCalc 9 (MedCalc Software, Mariakerke, Belgium).

110 patients with two consecutive visits, who, at the first visit, had well-controlled asthma, were included in the analysis. Of those patients, 61 lost control in the subsequent visit (32 with rhinitis and 29 without rhinitis) and 49 remained well

\section{TABLE 1 Patients' characteristics and loss of control}

\begin{tabular}{|c|c|c|c|c|}
\hline & Asthma without rhinitis & Asthma and rhinitis & Asthma without rhinitis & Asthma and rhinitis \\
\hline Subjects & 29 & 32 & 23 & 26 \\
\hline Age & $53 \pm 11$ & $50 \pm 18$ & $53 \pm 16$ & $48 \pm 16$ \\
\hline Sex male/female & $9 / 20$ & $11 / 21$ & $9 / 14$ & $10 / 16$ \\
\hline FEV $1 / F V C$ visit 1 & $74 \pm 10$ & $80 \pm 10$ & $78 \pm 7$ & $77 \pm 7$ \\
\hline FEV $_{1 / F V C}$ visit 2 & $72 \pm 14$ & $78 \pm 10$ & $72 \pm 14$ & $81 \pm 13$ \\
\hline FEF25-75 visit $1 \%$ pred & $65 \pm 31$ & $75 \pm 32$ & $72 \pm 38$ & $69 \pm 29$ \\
\hline FEF25-75 visit $2 \%$ pred & $59 \pm 30$ & $78 \pm 10$ & $60 \pm 30$ & $76 \pm 35$ \\
\hline$A C Q$ visit 1 & $0.76 \pm 0.43$ & $0.72 \pm 0.58$ & $0.69 \pm 0.48$ & $0.71 \pm 0.53$ \\
\hline
\end{tabular}

Data are presented as $n$ or mean \pm SD. *: $p<0.05$ compared to visit 1. FEV1: forced expiratory volume in $1 \mathrm{~s} ; \%$ pred: \% predicted; FVC: forced vital capacity; FEF25-75\%: forced expiratory flow at $25-75 \%$ of FVC; ACQ: Asthma Control Questionnaire; FeNO: exhaled NO fraction. 


\begin{tabular}{|c|c|c|c|c|c|c|c|c|}
\hline \multirow[t]{2}{*}{ TABLE 2} & \multicolumn{8}{|c|}{$\begin{array}{l}\text { Diagnostic performance of change in exhaled NO fraction for the identification of loss of control in two consecutive } \\
\text { visits }\end{array}$} \\
\hline & & $\begin{array}{l}\text { Optimal cut-off } \\
\text { point }\end{array}$ & $\begin{array}{l}\text { Sensitivity } \\
(95 \% \mathrm{Cl})\end{array}$ & $\begin{array}{l}\text { Specificity } \\
(95 \% \mathrm{Cl})\end{array}$ & PPV & NPV & $\begin{array}{c}\text { AUC } \\
(95 \% \mathrm{Cl})\end{array}$ & p-value \\
\hline \multirow{2}{*}{\multicolumn{2}{|c|}{ Asthma without rhinitis" }} & $>30 \%$ & $0.83(0.64-0.94)$ & $0.87(0.68-0.97)$ & 0.89 & 0.81 & $0.893(0.777-0.961)$ & $<0.0001$ \\
\hline & & $>40 \%$ & $0.72(0.53-0.86)$ & $0.92(0.74-0.98)$ & 0.92 & 0.71 & $0.786(0.657-0.884)$ & $<0.0001$ \\
\hline
\end{tabular}

controlled (26 with rhinitis and 23 without rhinitis). Demographic characteristics are presented in table 1. Study groups did not differ in demographic and spirometric characteristics, FeNO levels or ACQ scores at baseline. In both groups where control was lost in visit 2 (with and without rhinitis), FeNO increased significantly $(\mathrm{p}<0.001$ for both comparisons). In contrast, patients who maintained control at visit 2 did not present significant differences in FeNO levels between visits $(p=0.100$ and $p=0.146$ for patients with and without allergic rhinitis, respectively).

In ROC analysis, $\Delta F$ eNO $\%$ was associated with loss of control, since both ROC curves differ significantly from 0.5 (fig. 1 , table 2). The diagnostic performance of $\Delta F \mathrm{eNO} \%$ did not differ significantly between patients without and with allergic rhinitis, despite a trend in favour of the former (AUC 0.893 versus $0.786 ; \mathrm{p}=0.152$ ). In patients without rhinitis, an increase in $\mathrm{FeNO}>30 \%$ from baseline was highly indicative of loss in asthma control (PPV 0.89), whereas an increase $<20 \%$ was unlikely to be related to loss of control (NPV 0.81). Additionally, an increase in $\mathrm{FeNO}>40 \%$ in patients with rhinitis was also highly suggestive of loss in asthma control (PPV 0.92). In contrast, an increase in $\mathrm{FeNO}<20 \%$ was not as

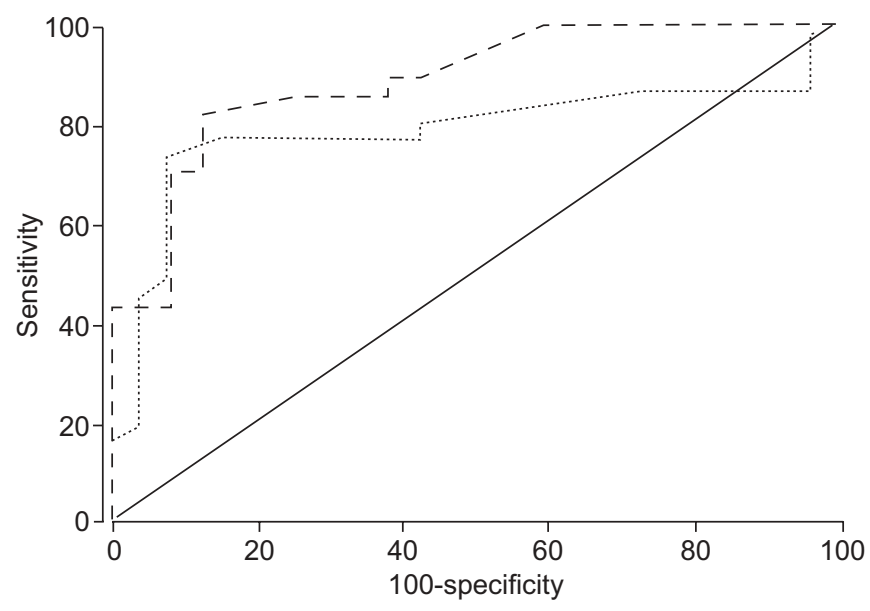

FIGURE 1. Receiver operating characteristic analysis for the assessment of the performance of exhaled NO fraction ( $\mathrm{FeNO}$ ) changes compared to baseline $\left(\Delta F_{\mathrm{eNO}} \%\right)$ in the identification of loss of control between two consecutive visits. - - - - : asthma without rhinitis; $\cdots \cdots \cdot \cdot$ : asthma and rhinitis. strong a predictor of maintenance of control (NPV 0.72) in patients with asthma and rhinitis as in those without rhinitis.

Our data support our previous observations suggesting that the presence of atopy, and especially allergic rhinitis, may impair the diagnostic value of FeNO in asthmatics [2]. The poorer diagnostic performance of $F$ eNO in patients with concomitant rhinitis may plausibly represent cases of deterioration in rhinitis symptoms (related to increased nasal inflammation) without concurrent impairment of asthma control. Interestingly, our results independently confirm the data of MichiLs et al. [1] and further support the observation that an increase in FeNO $>40 \%$ from baseline is highly indicative of loss of asthma control, even in patients with underlying atopy, as expressed by the presence of allergic rhinitis.

\section{A.I. Papaioannou, M. Minas, K. Tanou, K.I. Gourgoulianis and K. Kostikas}

Respiratory Medicine Dept, University of Thessaly Medical School, Larissa, Greece.

Correspondence: K. Kostikas, Respiratory Medicine Dept, University Hospital of Larissa, Larissa 41110, Greece. E-mail: ktk@otenet.gr

Statement of Interest: None declared.

\section{REFERENCES}

1 Michils A, Louis R, Peche R, et al. Exhaled nitric oxide as a marker of asthma control in smoking patients. Eur Respir J 2009; 33: 1295-1301.

2 Kostikas K, Papaioannou AI, Tanou K, et al. Portable exhaled nitric oxide as a screening tool for asthma in young adults during pollen season. Chest 2008; 133: 906-913.

3 Franklin PJ, Stick SM, Le Souef PN, et al. Measuring exhaled nitric oxide levels in adults: the importance of atopy and airway responsiveness. Chest 2004; 126: 1540-1545.

4 Juniper EF, Svensson K, Mork AC, et al. Measurement properties and interpretation of three shortened versions of the asthma control questionnaire. Respir Med 2005; 99: 553-558.

5 Tanou K, Koutsokera A, Kiropoulos TS, et al. Inflammatory and oxidative stress biomarkers in allergic rhinitis: the effect of smoking. Clin Exp Allergy 2009; 39: 345-353.

DOI: $10.1183 / 09031936.00065209$ 